\title{
Effects of Wenxin Keli on the Action Potential and L-Type Calcium Current in Rats with Transverse Aortic Constriction-Induced Heart Failure
}

\author{
Yu Chen, ${ }^{1,2}$ Yang Li, ${ }^{3}$ Lili Guo, ${ }^{2}$ Wen Chen, ${ }^{2}$ Mingjing Zhao, ${ }^{4}$ Yonghong Gao, ${ }^{4}$ \\ Aiming Wu, ${ }^{4}$ Lixia Lou, ${ }^{4}$ Jie Wang, ${ }^{2}$ Xiaoqiu Liu, ${ }^{1}$ and Yanwei Xing ${ }^{2}$ \\ ${ }^{1}$ Shenyang Pharmaceutical University, Shenyang, Liaoning 110016, China \\ ${ }^{2}$ Guang'anmen Hospital, China Academy of Chinese Medical Sciences, Beijing 100053, China \\ ${ }^{3}$ Institute of Geriatric Cardiology of Chinese PLA General Hospital, Beijing 100853, China \\ ${ }^{4}$ The Key Laboratory of Chinese Internal Medicine of the Ministry of Education, Dongzhimen Hospital Affiliated to Beijing, \\ University of Chinese Medicine, Beijing 100700, China
}

Correspondence should be addressed to Xiaoqiu Liu; liuxiaoqiu3388@tom.com and Yanwei Xing; xingyanwei12345@163.com

Received 21 March 2013; Revised 8 September 2013; Accepted 10 September 2013

Academic Editor: Hao Xu

Copyright @ $2013 \mathrm{Yu}$ Chen et al. This is an open access article distributed under the Creative Commons Attribution License, which permits unrestricted use, distribution, and reproduction in any medium, provided the original work is properly cited.

Objective. We investigated the effects of WXKL on the action potential (AP) and the L-type calcium current $\left(I_{\mathrm{Ca}-\mathrm{L}}\right)$ in normal and hypertrophied myocytes. Methods. Forty male rats were randomly divided into two groups: the control group and the transverse aortic constriction- (TAC-) induced heart failure group. Cardiac hypertrophy was induced by TAC surgery, whereas the control group underwent a sham operation. Eight weeks after surgery, single cardiac ventricular myocytes were isolated from the hearts of the rats. The APs and $I_{\mathrm{Ca}-\mathrm{L}}$ were recorded using the whole-cell patch clamp technique. Results. The action potential duration (APD) of the TAC group was prolonged compared with the control group and was markedly shortened by WXKL treatment in a dose-dependent manner. The current densities of the $I_{\mathrm{Ca}-\mathrm{L}}$ in the TAC group treated with $5 \mathrm{~g} / \mathrm{L}$ WXKL were significantly decreased compared with the TAC group. We also determined the effect of WXKL on the gating mechanism of the $I_{\mathrm{Ca}-\mathrm{L}}$ in the TAC group. We found that WXKL decreased the $I_{\mathrm{Ca}-\mathrm{L}}$ by accelerating the inactivation of the channels and delaying the recovery time from inactivation. Conclusions. The results suggest that WXKL affects the AP and blocked the $I_{\mathrm{Ca}-\mathrm{L}}$, which ultimately resulted in the treatment of arrhythmias.

\section{Introduction}

Cardiovascular diseases (CVD) are the most common threat to human health worldwide. From 1999 to 2009, the relative rates of death attributable to CVD decreased by $32.7 \%$. However, CVD still accounted for $32.3 \%$ of deaths in the United States in 2009. Thus, the burden of this disease remains high. Epidemiological data show that an estimated 5.1 million Americans greater than 20 years of age exhibited heart failure from 2007 to 2010. Projections show that the prevalence of heart failure by 2030 will increase by $25 \%$ from the 2013 estimates [1]. In many cardiovascular diseases, cardiac hypertrophy is a common pathological process, and the disorder of the heart rhythm that is induced by cardiac hypertrophy is the most common cause of sudden cardiovascular death. A previous study indicates that $36 \%$ of the 690 athletes died as a result of cardiac hypertrophy [2]. This high incidence of cardiac hypertrophy has caused widespread concern, and these data highlight the importance of finding suitable drugs for treating cardiac hypertrophyinduced arrhythmias.

The currently electrophysiological research on cardiac hypertrophy mainly focuses on changes in the action potentials and the related ionic mechanisms. In recent years, the role of Ca signalling in cardiac myocytes was studied with respect to electrophysiology, such as the effect of Ca signalling on arrhythmias and action potentials [3]. $\mathrm{Ca}^{2+}$ enters the cell via the L-type $\mathrm{Ca}^{2+}$ current, and the sarcoplasmic reticulum 
(SR) releases $\mathrm{Ca}^{2+}$ via the ryanodine receptor (RyR) to increase the intracellular calcium concentration $\left(\left[\mathrm{Ca}^{2+}\right] \mathrm{i}\right)$ and thus activate cardiac contraction. However, the overloading of the SR with $\mathrm{Ca}^{2+}$ can induce arrhythmias [4]. Blocking $\mathrm{Ca}^{2+}$ channel and reducing the $\mathrm{Ca}^{2+}$ overload will thus benefit the treatment of arrhythmias and heart failure. The most important finding in animal models of left ventricular hypertrophy is the significant prolongation of the action potential duration (APD) at low but not at high frequencies. Recent model results suggest that even subtle changes in AP morphology that may result from remodeling of membrane transporter expression in disease may have major impact on the temporal waveform of $\mathrm{Ca}^{2+}$ transients, thus influencing tissue level electromechanical function [5]. The prolongation of the APD would increase the incidence of triggered activity and the early after-depolarization (EAD), which would result in increasing the incidence of arrhythmias [6,7]. Using ion channel antagonist drugs to treat arrhythmias in patients with structural heart disease does not reduce their mortality $[8,9]$. In addition, most antiarrhythmic drugs have shown potential lethal proarrhythmic effects that result in the aggravation of arrhythmias and potentially induce ventricular arrhythmias. Thus, there is an urgent need to develop effective and safer antiarrhythmic agents. In particular, traditional Chinese medicine has been well recognized for its antiarrhythmic potential.

Wenxin Keli (WXKL) is a Chinese herb extract developed by Guanganmen Hospital at the Chinese Academy of Chinese Medical Sciences and is the first antiarrhythmic Chinese medicine to be approved by the state. It is composed of five main components: Nardostachys chinensis Batal extract, Codonopsis, Notoginseng, amber, and Rhizoma Polygonati. Since its clinical application, this drug had been proven to be of benefit in the treatment of various diseases, such as cardiac arrhythmias, cardiac inflammation, and chronic heart failure [10]. Previous studies have shown that WXKL is a safe and effective treatment, which does not result in significant adverse reactions for the premature ventricular contractions caused by viral myocarditis, as evaluated by improvements in the clinical symptoms, signs, and ECG [11]. Some studies have investigated the effects of irbesartan, amiodarone, and WXKL, applied either alone or in combination, on sinus rhythm maintenance in patients with atrial fibrillation after conversion. It was shown that a combined therapy of Chinese and Western medicines exhibits a synergistic antiarrhythmia effect that improves the conversion rate of atrial fibrillation, shortens the conversion time, and avoids adverse reaction [12, 13]. A fascinating electrophysiologic study of the effects of WXKL suggests that this agent can depress the sodium channel-dependent parameters in canine isolated coronary-perfused preparations and effectively manages and prevents the induction of atrial fibrillation [14]. In addition, the action of notoginseng, which is one of the components of WXKL, was demonstrated to have antiarrhythmic properties, as demonstrated in rats with ischemia arrhythmias [15]. As a result, notoginseng further enhances the antiarrhythmic properties of WXKL. Moreover, it has been reported that WXKL exhibits beneficial effects on isoproterenol- (ISO-) induced heart failure in rats. In addition, WXKL can greatly improve ISO-induced cardiac dysfunction and protect against aconitine-induced arrhythmia in rats [16]. A recent study suggested that long-term treatment with WXKL may have seen it attenuate ischemiainduced ventricular arrhythmias in rats and inhibit $I_{\mathrm{Ca}-\mathrm{L}}$ and $I_{t o}$ in a concentration-dependent manner, which may contribute to the observed attenuation [17].

Although a number of electrophysiological studies have analysed the use of WXKL for the treatment of cardiac hypertrophy, the effects of WXKL on the APs and $I_{\mathrm{Ca}-\mathrm{L}}$ of normal and hypertrophied myocytes have not been reported. Thus, the goal of this study was to evaluate the effect of WXKL on the APs and $I_{\mathrm{Ca}-\mathrm{L}}$ of normal and hypertrophic ventricular myocytes using whole-cell patch clamp recording techniques and to explore the mechanism through which WXKL benefits the treatment of cardiac hypertrophy, which would provide better insights into the effects of antiarrhythmic drugs.

\section{Materials and Methods}

2.1. Animals. Forty male Sprague-Dawley rats (body weight $=140-160 \mathrm{~g}$ ), which were purchased from Vital River Experimental Animal Centre (license number SYXK (E) 200420007, Beijing, China) were randomly divided into two groups: the TAC group $(n=25)$ and the control group $(n=15)$. The TAC rats underwent transverse aortic constriction (TAC) surgery, and the control group underwent an identical procedure but without the application of the ligation. All of the following experiments conformed to the Guiding Principles for the Care and Use of Laboratory Animals issued by the National Committee of Science and Technology of China.

2.2. Drugs and Solutions. WXKL was provided by Shandong Buchang Pharmaceuticals Co., Ltd., China. The drug was dissolved with $\mathrm{Ca}^{2+}$-free Tyrode solution at concentrations of $0.5 \mathrm{~g} / \mathrm{L}, 1 \mathrm{~g} / \mathrm{L}, 5 \mathrm{~g} / \mathrm{L}, 10 \mathrm{~g} / \mathrm{L}$, and $20 \mathrm{~g} / \mathrm{L}$ prior to the experiment. The $\mathrm{Ca}^{2+}$-free Tyrode solution contained (in mmol/L) $137 \mathrm{NaCl}, 5.4 \mathrm{KCl}, 1.0 \mathrm{MgCl}_{2}, 0.33 \mathrm{NaH}_{2} \mathrm{PO}_{4}, 10$ hydroxyethyl piperazine ethanesulfonic acid (HEPES), and 10 glucose ( $\mathrm{pH}$ 7.35, adjusted with $\mathrm{NaOH}$ ). The Krebs buffer (KB) solution for cell storage contained (in $\mathrm{mmol} / \mathrm{L}$ ) $40 \mathrm{KCl}, 20 \mathrm{KH}_{2} \mathrm{PO}_{4}$, $3.0 \mathrm{MgCl}_{2}, 70 \mathrm{KOH}, 50 \mathrm{~L}$-glutamic acid, 10 HEPES, 20 taurine, 10 glucose, and $0.5 \mathrm{EGTA}$ (pH 7.35, adjusted with $\mathrm{KOH}$ ). For the AP recordings, the internal pipette solution contained (in $\mathrm{mmol} / \mathrm{L}$ ) $120 \mathrm{~K}$ aspartate, $20 \mathrm{KCl}$, $1.0 \mathrm{MgCl}_{2}, 4.0 \mathrm{Na}_{2} \mathrm{ATP}$, 10 glucose, and 10 HEPES, and the bath solution contained (in $\mathrm{mmol} / \mathrm{L}) 140 \mathrm{NaCl}, 1.0 \mathrm{CaCl}_{2}, 1.0 \mathrm{MgCl}_{2}, 4 \mathrm{KCl}, 10$ HEPES, and 5 glucose. For the $I_{\mathrm{Ca}-\mathrm{L}}$ recordings, the internal pipette solution contained (in mmol/L) $120 \mathrm{CsCl}, 1.0 \mathrm{CaCl}_{2}, 5.0$ $\mathrm{MgCl}_{2}, 5.0 \mathrm{Na}_{2}$ ATP, 11 EGTA, 10 HEPES, and 11 glucose ( $\mathrm{pH} 7.3$, adjusted with $\mathrm{CsOH}$ ), and the bath solution was the Tyrode solution supplemented with $1.8 \mathrm{mmol} / \mathrm{L} \mathrm{CaCl}_{2}$.

2.3. Creation of the TAC Model. The transverse aortic constriction (TAC) surgery was performed in male SpragueDawley rats as described previously $[18,19]$. Briefly, the rats were anesthetised with $3 \%$ chloral hydrate $(300 \mathrm{mg} / \mathrm{kg}$, 
intraperitoneally). The thorax was opened, and a 4-0 silk suture was passed under the aorta between the origin of the right innominate and the left common carotid arteries. A $6 \mathrm{G}$ needle was placed on the ascending aorta, and the suture was snugly tied around the needle and the aorta. The probe was then quickly removed. The skin was closed, and the rats were maintained in a heating pad until they recovered from the anesthesia. The sham-operated animals underwent an identical procedure but without the application of the ligation. After surgery, both groups were fed tap water and normal fodder in different cages for 8 weeks. To characterise the model, echocardiographic measurements were obtained 8 weeks after surgery using a Vivid 7 Dimension cardiovascular ultrasound system (GE Healthcare, Fairfield, Connecticut, United States) as described previously [20]. The detection indicator was the left ventricular posterior wall thickness (LPWD), and the parameters measured are shown in Figure 1(e).

2.4. Cardiac Ventricular Myocytes Isolation. Single cardiac ventricular myocytes were isolated from the hearts of the rats as previously described [21] with slight modifications. Briefly, 5 minutes after the rats were heparinised $(100 \mathrm{U} / \mathrm{mL}$ $1 \mathrm{~mL} / 100 \mathrm{~g}$ i.p.), the animals were anesthetised with $3 \%$ chloral hydrate $(0.5 \mathrm{~mL} / 100 \mathrm{~g}$ i.p). The heart was rapidly excised and mounted on the Langendorff apparatus and perfused via the aorta with oxygenated $\mathrm{Ca}^{2+}$-free Tyrode solution for 5 minutes and then with $\mathrm{Ca}^{2+}$-free Tyrode solution containing collagenase II $(0.6 \mathrm{mg} / \mathrm{mL}$, Worthington, USA), trypsin ( $0.24 \mathrm{mg} / \mathrm{mL}$, Amresco, USA), and proteinase E $\left(0.08 \mathrm{mg} / \mathrm{mL}\right.$, Amresco, USA) for $15-20$ minutes at $37^{\circ} \mathrm{C}$. Subsequently, the ventricular tissue was excised, cut into small pieces in a dish containing KB solution, and blown gently to obtain single ventricular myocytes. The cells were maintained at $4^{\circ} \mathrm{C}$ in $\mathrm{KB}$ solution until use. All of the solutions were continuously gassed with $95 \% \mathrm{O}_{2}$ and $5 \%$ $\mathrm{CO}_{2}$ at $37^{\circ} \mathrm{C}$. The single ventricular myocyte selected for electrophysiological measurements is rod-shaped, quiescent, Ca-tolerant, and had clear cross-striations and a smooth and glossy surface.

2.5. Histological Examination. The rat heart samples were cut into transverse sections and routinely stained with haematoxylin and eosin (H\&E) as described previously [22]. The stained sections were examined under a light microscope (OLYMPUS BX51, Japan) and photographed at 400x magnification for morphological analysis.

2.6. Electrophysiological Recording. The whole-cell patch clamp technique was used to record the APs and $I_{\text {Ca-L }}$ using an Axopatch 700B amplifier (Axon Instruments, USA) with the pCLAMP 9.2 software (Axon Instruments, USA). Borosilicate glass patch pipettes (resistance $=3-5 \mathrm{M} \Omega$ ) were pulled using a vertical pipette puller (Narishige pp-830, Japan). The cells were maintained in external solution for 5 to 10 minutes after perfusion and the data were recorded after entering the cell for 5 minutes to stabilise the current. All of the recordings were performed at room temperature $\left(22^{\circ} \mathrm{C}\right)$ within 25 minutes to avoid current rundown. The APs were elicited in the current-clamp mode at a rate of $1.0 \mathrm{~Hz}$ using 30 trains of suprathreshold current pulses. The membrane capacitance was calculated using the manual whole-cell capacitance controls on the Axopatch amplifier. The $I_{\text {Ca-L }}$ was recorded in the voltage-clamp mode and elicited through step depolarisation from -40 to $+50 \mathrm{mV}$ in $10-\mathrm{mV}$ increments for $250 \mathrm{~ms}$.

2.7. Statistical Analysis. Off-line leak correction was performed on all of the amplitude data. The pCLAMP 9.2 software (Axon Instruments, USA) and the Origin 6.1 software (Microcal Software, USA) were used for the data acquisition and analysis. The data are presented as the mean $\pm \mathrm{SE}$, where $n$ represents the number of cells analysed. The statistical comparisons between different groups were performed with ANOVA and Student's $t$-test. Differences with a value of $P$ less than 0.05 were considered statistically significant.

\section{Results}

3.1. Echocardiographic and Histological Characteristics. Eight weeks after the TAC surgery, the cardiac structure and function were measured through echocardiographic and histological examinations. Compared with the control group, the heart was slightly enlarged (Figure 1(a)), and the wall of ventricle was thickened (Figure 1(c)) in the TAC group. A significant difference was found in the left ventricular apical biopsy between the control group and the TAC group (Figure 1(b), HE staining 400x magnification). The single ventricular myocytes in the TAC group were larger than those in the control group (Figure 1(d)). We evaluated the cardiac systolic and diastolic functions by measuring the left ventricular posterior wall thickness (LPWD) of the control group and the TAC group (Figure 1(e)). Compared with the control group, the LPWD of the TAC group was significantly increased $(0.22 \pm 0.02 \mathrm{~cm}$ versus $0.31 \pm 0.03 \mathrm{~cm}, n=10$, $P<0.01$, Figure 1(e3)).

3.2. Effects of WXKL on the APs in the Control Group and the TAC Group. The APs were recorded by applying a $900-\mathrm{pA}$ current pulse with duration of $3 \mathrm{~ms}$ at $1 \mathrm{~Hz}$ in the currentclamp mode. The APD was significantly prolonged in the TAC group compared with the control group (Figure 2(a)). The APDs obtained with 20\%, 50\%, and 90\% repolarisation $\left(\mathrm{APD}_{20}, \mathrm{APD}_{50}\right.$, and $\mathrm{APD}_{90}$ in ms) in the control group and the TAC group were the following: $48.5 \pm 3.5 \mathrm{~ms}$ versus $81.9 \pm$ $4.3 \mathrm{~ms}(n=6, P<0.01), 98.7 \pm 8.8 \mathrm{~ms}$ versus $137.2 \pm 13.4 \mathrm{~ms}$ $(n=6, P<0.01)$, and $142.0 \pm 9.3 \mathrm{~ms}$ versus $163.7 \pm 2.9 \mathrm{~ms}$ $(n=6, P<0.05)$, respectively (Figure 2(b)).

After treatment with different dose of WXKL, the APD in the TAC group exhibited significant changes (Figure 2(c)). The $\mathrm{APD}_{20}, \mathrm{APD}_{50}$, and $\mathrm{APD}_{90}$ in the TAC group were markedly shortened by WXKL in a dose-dependent manner. After perfusion with $1 \mathrm{~g} / \mathrm{L} \mathrm{WXKL}$, the $\mathrm{APD}_{20}, \mathrm{APD}_{50}$, and $\mathrm{APD}_{90}$ were shortened from $81.9 \pm 4.3 \mathrm{~ms}$ to $67.4 \pm 6.2 \mathrm{~ms}$ $(n=6, P<0.05), 137.2 \pm 13.4 \mathrm{~ms}$ to $122.8 \pm 11.6 \mathrm{~ms}$ $(n=6, P<0.05)$, and $163.7 \pm 2.9 \mathrm{~ms}$ to $144.4 \pm 3.2 \mathrm{~ms}$ 


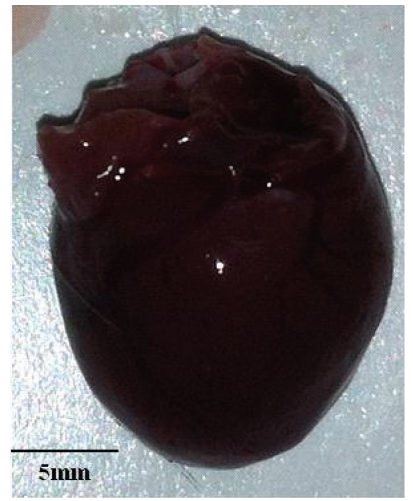

(a)

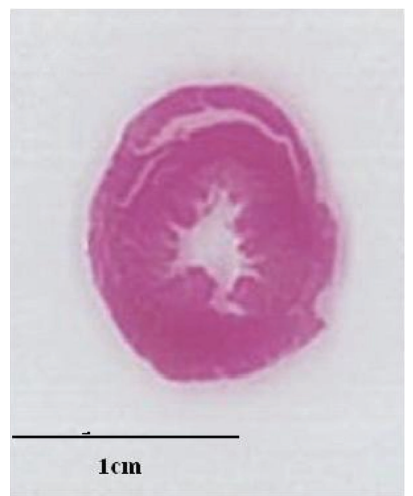

(c)

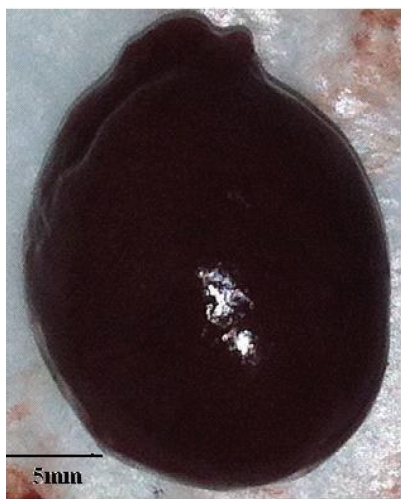

c2

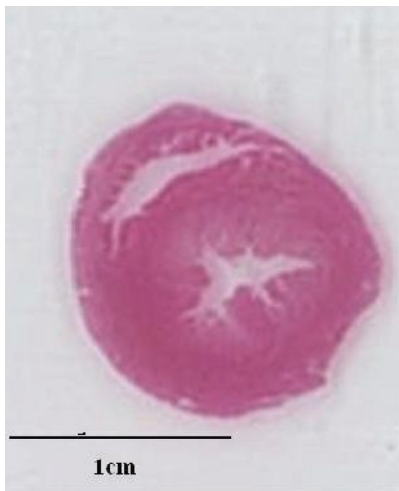

e1
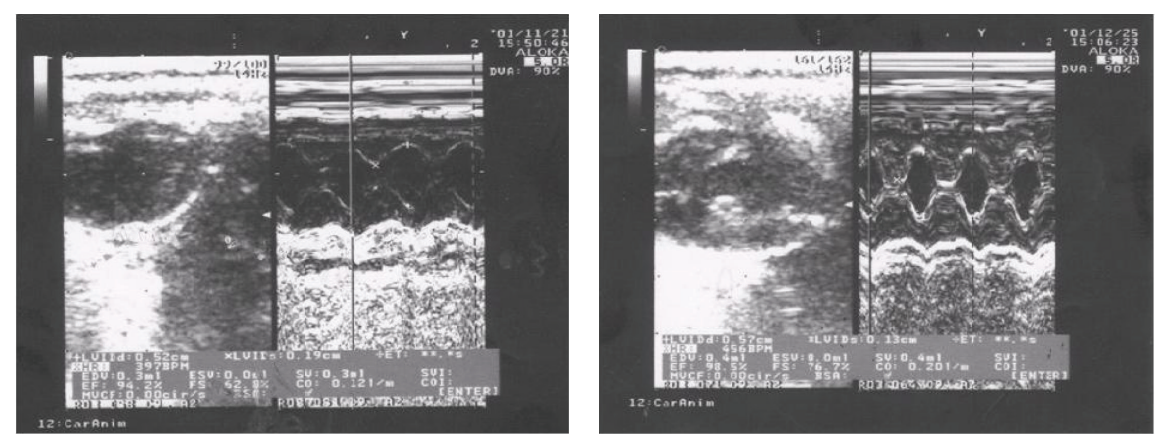

b1

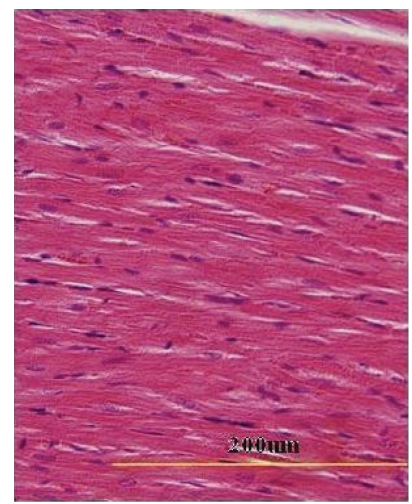

(b)

$\mathrm{d} 1$

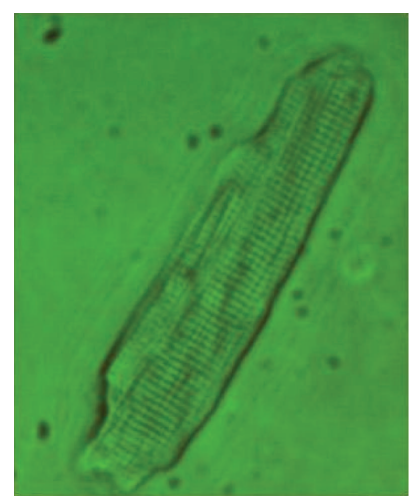

b2
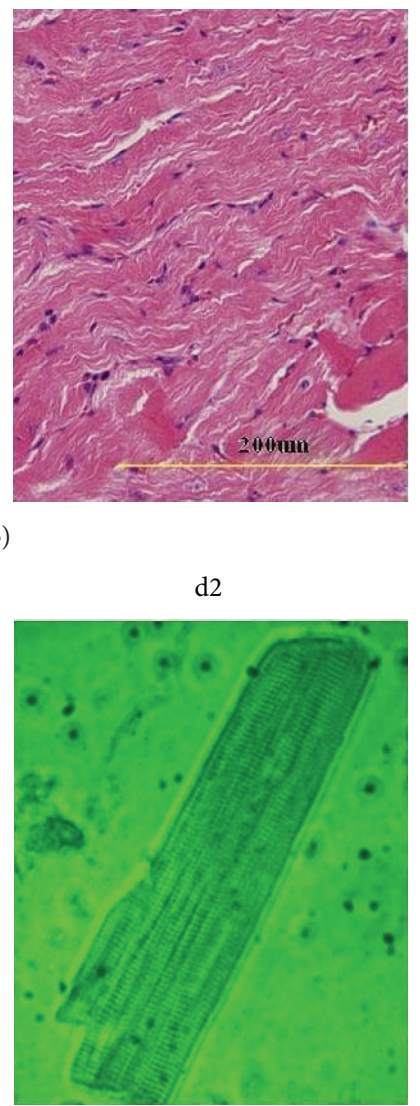

(d)

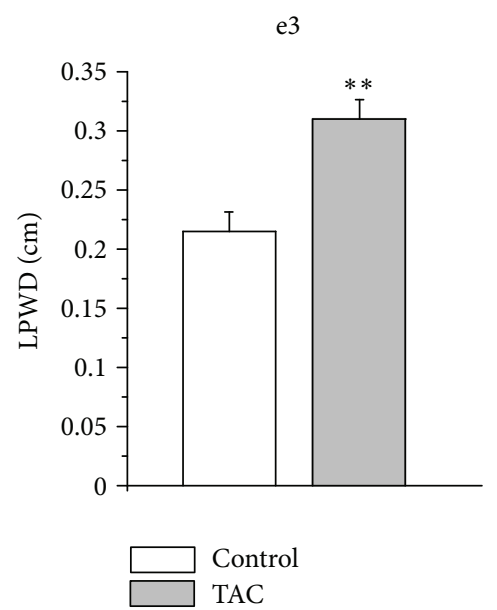

(e)

FIGURE 1: (a) Preparation of the hearts from the control group (a1) and the TAC group (a2). (b) Left ventricular apical biopsy of the control group ((b1), HE staining 400x magnification) and the TAC group ((b2), HE staining 400x magnification). (c) Pathological section of the largest cross-section of the control group (c1) and the TAC group (c2). (d) Single ventricular myocytes from the control group (d1) and the TAC group (d2). (e) Typical echocardiography images from the control group (e1) and the TAC group (e2). Eight weeks after the TAC surgery, the cardiac structure and function were measured through echocardiography. We evaluated the cardiac systolic and diastolic functions by measuring the left ventricular posterior wall thickness (LPWD) of the control group and the TAC group (c3). ${ }^{* *} P<0.01$ versus the control group. 


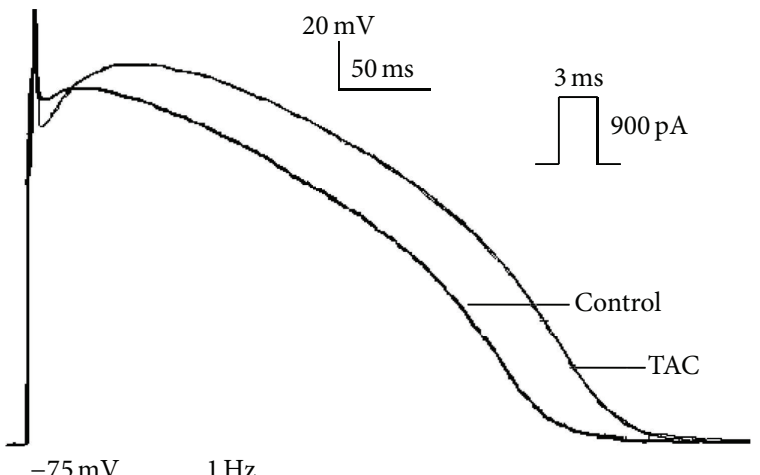

(a)

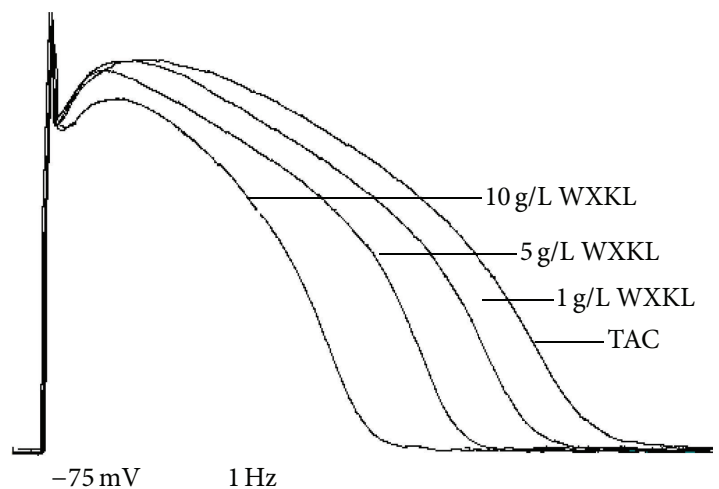

(c)

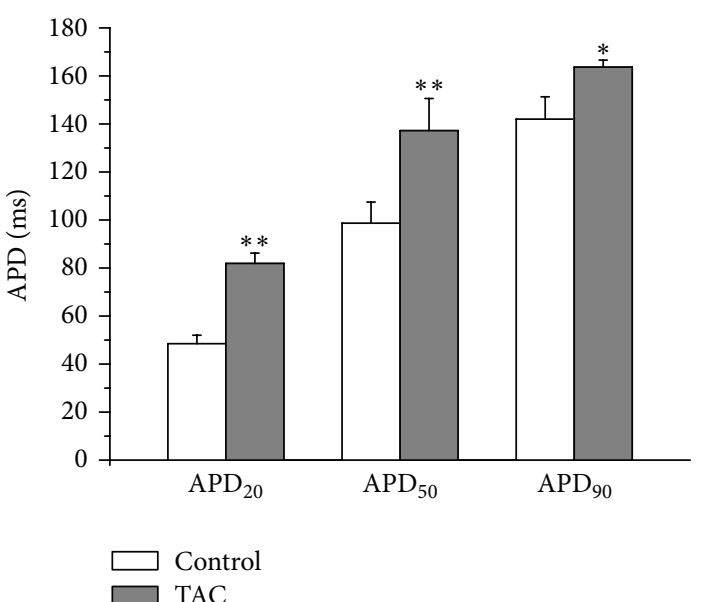

(b)

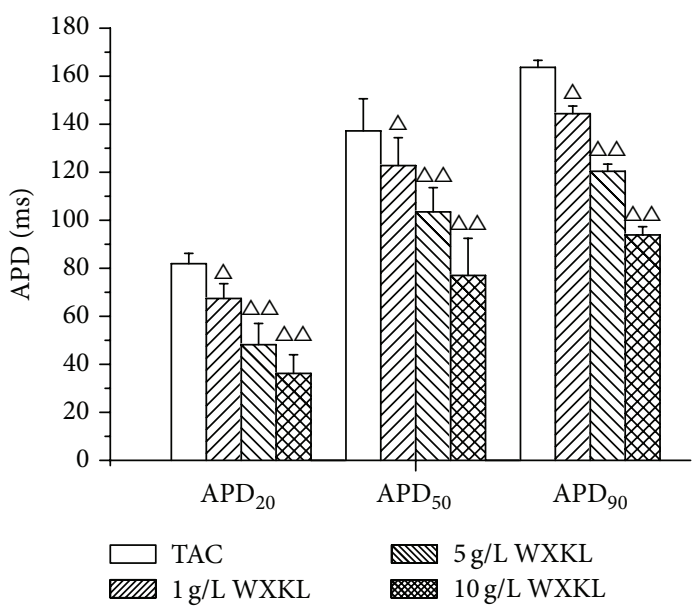

(d)

FIGURE 2: Representative AP traces recorded from the control and the TAC group and effects of different concentrations of WXKL on the APs in the TAC group. (a) APs of the control group and the TAC group. (b) $\mathrm{APD}_{20}, \mathrm{APD}_{50}$, and $\mathrm{APD}_{90}$ of the control group and the TAC group. (c) Effects of 1, 5, and $10 \mathrm{~g} / \mathrm{L}$ WXKL on the APs in the TAC group. (d) $\mathrm{APD}_{20}, \mathrm{APD}_{50}$, and $\mathrm{APD}_{90}$ of the TAC group after treatment with 1,5 , and $10 \mathrm{~g} / \mathrm{L}$ WXKL. ${ }^{*} P<0.05$ and ${ }^{* *} P<0.01$ versus the control group. ${ }^{\Delta} P<0.05$ and ${ }^{\Delta \Delta} P<0.01$ versus the TAC group.

( $n=6, P<0.05)$, respectively. When treated with $5 \mathrm{~g} / \mathrm{L}$ WXKL, the $\mathrm{APD}_{20}, \mathrm{APD}_{50}$, and $\mathrm{APD}_{90}$ were shortened from $81.9 \pm 4.3 \mathrm{~ms}$ to $48.2 \pm 8.8 \mathrm{~ms}(n=6, P<0.01), 137.2 \pm 13.4 \mathrm{~ms}$ to $103.5 \pm 10.1 \mathrm{~ms}(n=6, P<0.01)$, and $163.7 \pm 2.9 \mathrm{~ms}$ to $120.4 \pm 3.0 \mathrm{~ms}(n=6, P<0.01)$, respectively. When treated with $10 \mathrm{~g} / \mathrm{L}$ WXKL, the $\mathrm{APD}_{20}, \mathrm{APD}_{50}$, and $\mathrm{APD}_{90}$ were shortened from $81.9 \pm 4.3 \mathrm{~ms}$ to $36.2 \pm 7.8 \mathrm{~ms}(n=6$, $P<0.01), 137.2 \pm 13.4 \mathrm{~ms}$ to $77.0 \pm 15.5 \mathrm{~ms}(n=6, P<0.01)$, and $163.7 \pm 2.9 \mathrm{~ms}$ to $93.9 \pm 3.4 \mathrm{~ms}(n=6, P<0.01)$, respectively (Figure $2(\mathrm{~d})$ ). To understand the mechanisms that were responsible for the observed changes in the APD in the TAC group, we examined the $I_{\mathrm{Ca}-\mathrm{L}}$ in the control group and the TAC group.

3.3. Effects of WXKL on the $I_{C a-L}$ in the Control Group. To avoid the influence of current rundown, all of the recordings were obtained within 25 minutes. The current traces were obtained during a depolarising pulse from the holding potential of $-40 \mathrm{mV}$ to $0 \mathrm{mV}$ over $250 \mathrm{~ms}$. After WXKL $(0.5,1,5,10$, and $20 \mathrm{~g} / \mathrm{L}$ ) treatment, the $I_{\mathrm{Ca}-\mathrm{L}}$ in control group significantly decreased by $27.45 \pm 2.51 \%, 40.57 \pm 1.77 \%, 48.15 \pm 1.95 \%$, $58.22 \pm 2.96 \%$, and $71.68 \pm 2.63 \%$, respectively $(n=10$, $P<0.01$, Figure 3(a)). Thus, we concluded that WXKL significantly decreased the $I_{\mathrm{Ca}-\mathrm{L}}$ of the control group in a concentration-dependent manner. The $\mathrm{IC}_{50}$ of WXKL was found to be $6.23 \mathrm{~g} / \mathrm{L}$ (Figure 3(b)). The time-dependent curve showed that the effects of WXKL on the $I_{\mathrm{Ca}-\mathrm{L}}$ remained stable after 5 minutes (Figure 3(c)).

3.4. Effects of WXKL on the $I_{C a-L}$ in the TAC Group. The current-voltage $(I-V)$ curves were obtained by applying voltage steps in $10-\mathrm{mV}$ increments $(-40 \mathrm{mV}$ to $+50 \mathrm{mV})$ for $250 \mathrm{~ms}$ from a holding potential of $-40 \mathrm{mV}$. The representative $I_{\mathrm{Ca}-\mathrm{L}}$ traces show that the amplitude of the $I_{\mathrm{Ca}-\mathrm{L}}$ was higher in the TAC group compared with the control group (Figures 4(a) and 4(b)). After treatment with $5 \mathrm{~g} / \mathrm{L}$ WXKL, the $I_{\text {Ca-L }}$ was significantly reduced in the TAC group (Figure $4(\mathrm{c}))$. The $I-V$ curves also show that the current 


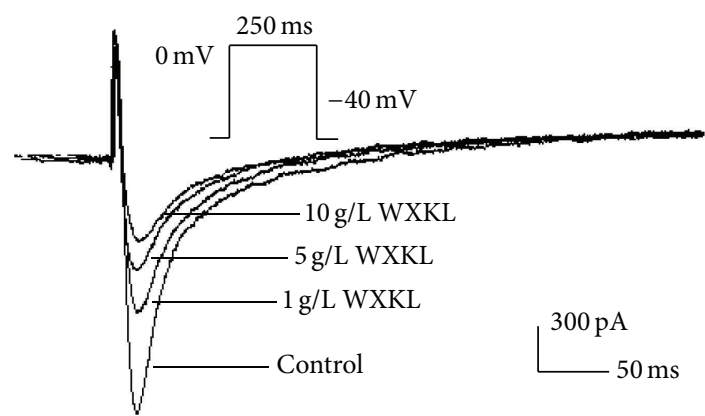

(a)

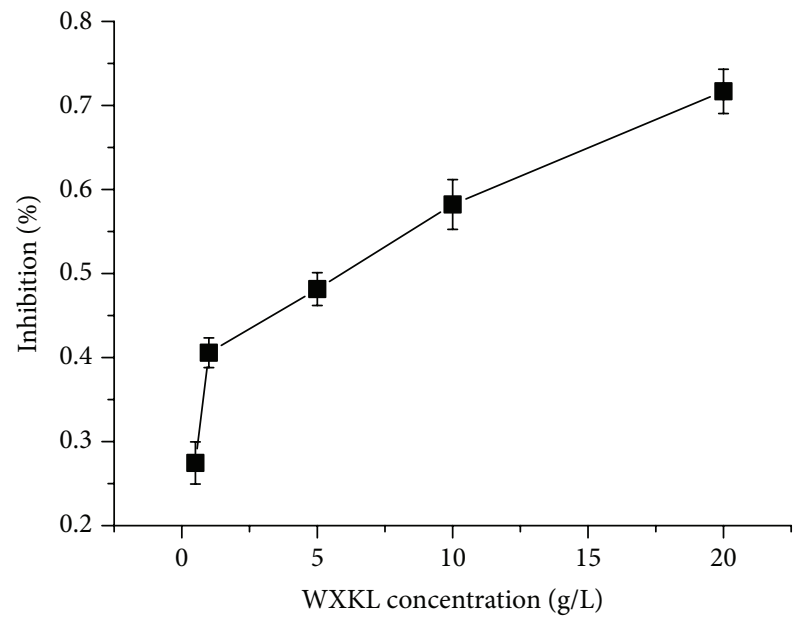

(b)

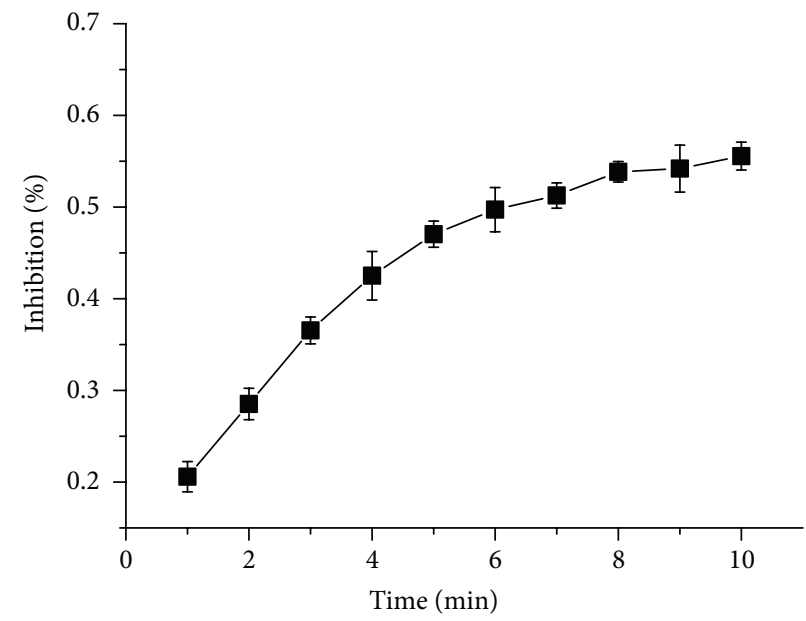

(c)

FIgURE 3: Effects of WXKL on the $I_{\mathrm{Ca}-\mathrm{L}}$ in the control group. (a) Effects of 1,5 , and $10 \mathrm{~g} / \mathrm{L}$ WXKL on the $I_{\mathrm{Ca}-\mathrm{L}}$ in the control group. After treatment with WXKL, the current amplitudes of the control group were significantly reduced. (b) Concentration-dependent effects of WXKL on the $I_{\mathrm{Ca}-\mathrm{L}}$ in the control group $\left(\mathrm{IC}_{50}=6.23 \mathrm{~g} / \mathrm{L}\right)$. (c) Time-dependent effects of $5 \mathrm{~g} / \mathrm{L}$ WXKL on the $I_{\mathrm{Ca}-\mathrm{L}}$ in the control group. ${ }^{*} P<0.05$ and ${ }^{* *} P<0.01$ versus the control group.

densities in the TAC group were significantly increased by a range of $-10 \mathrm{mV}$ to $+10 \mathrm{mV}$ compared with the control group $(n=10, P<0.01)$. The current densities of the TAC group after treatment with $5 \mathrm{~g} / \mathrm{L}$ WXKL were significantly decreased by a range of $-10 \mathrm{mV}$ to $+40 \mathrm{mV}$ compared with untreated TAC group $(n=10, P<0.01$, Figure $4(\mathrm{~d}))$. The mean current densities at $0 \mathrm{mV}$ were $-8.56 \pm 0.20 \mathrm{pA} / \mathrm{pF}$ in the control group and $-9.52 \pm 0.40 \mathrm{pA} / \mathrm{pF}$ in the TAC group $(n=10$, $P<0.05$, Figure $4(e))$. In contrast, the mean current density at $0 \mathrm{mV}$ in the TAC group after treatment with $5 \mathrm{~g} / \mathrm{L}$ WXKL was decreased to $-5.86 \pm 0.69 \mathrm{pA} / \mathrm{pF}(n=10, P<0.01$, Figure 4(e)).

3.5. Effects of WXKL on the Steady-State Activation and Inactivation Kinetics of $I_{\mathrm{Ca}-\mathrm{L}}$ in the TAC Group. The steadystate activation curves of $I_{\mathrm{Ca}-\mathrm{L}}$ were determined using pulses from $-40 \mathrm{mV}$ to $+50 \mathrm{mV}$ at $10-\mathrm{mV}$ increments for $250 \mathrm{~ms}$. The steady-state inactivation curves of $I_{\mathrm{Ca}-\mathrm{L}}$ were determined using pulses from $-60 \mathrm{mV}$ to $+30 \mathrm{mV}$ at $10-\mathrm{mV}$ increments for $1,000 \mathrm{~ms}$. The steady-state activation curves was described assuming that a Boltzmann function:

$$
\left(\frac{G}{G_{\max }}=\frac{1}{1+\exp \left(\left(V_{1 / 2, \text { ack }}-V_{\text {rev }}\right) / k\right)}\right) .
$$

The steady-state inactivation curves was described assuming that a Boltzmann function:

$$
\left(\frac{I}{I_{\max }}=\frac{1}{1+\exp \left(\left(V_{\text {rev }}-V_{1 / 2, \text { inact }}\right) / k\right)}\right) .
$$

The steady-state activation curve in each group exhibited no significant difference (Figure 5(a)). The half-activation potentials $\left(V_{1 / 2 \text {,act }}\right.$, at which $50 \%$ of the channels are activated) in the control and the TAC groups were $12.01 \pm 0.91 \mathrm{mV}$ and $13.55 \pm 1.55 \mathrm{mV}$, respectively $(n=10, P>0.05)$. Treatment 


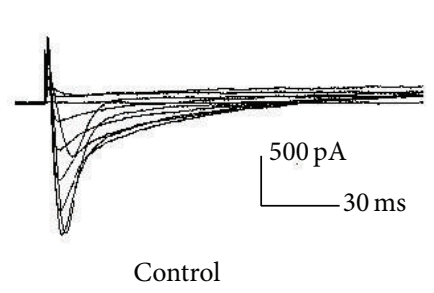

(a)

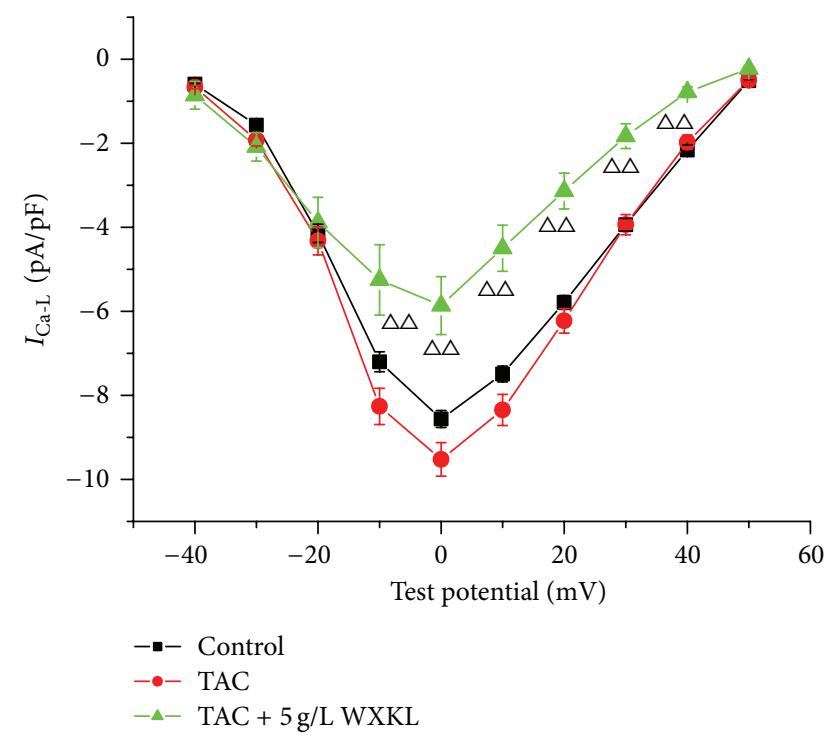

(d)

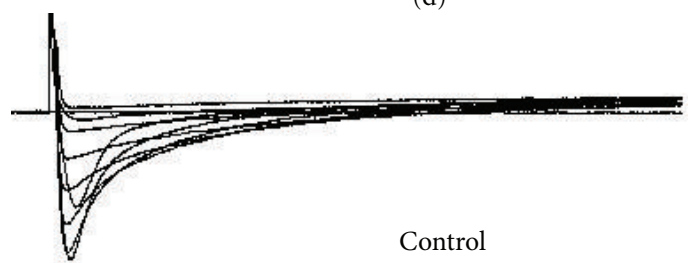

(f)

(b)
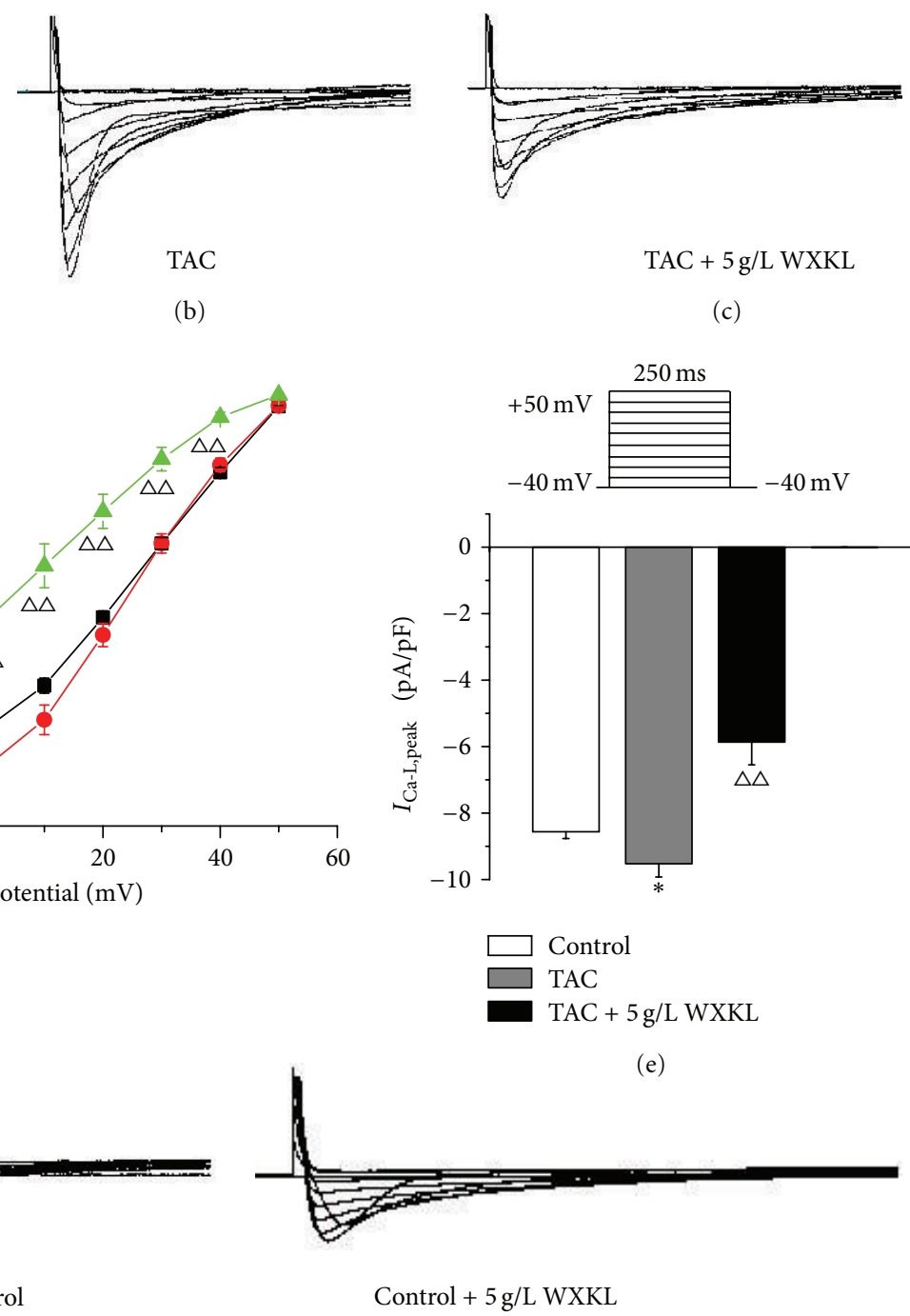

(g)

FIGURE 4: Effects of WXKL on the $I_{\mathrm{Ca}-\mathrm{L}}$ in the TAC group. (a) Representative $I_{\mathrm{Ca}-\mathrm{L}}$ traces recorded from the control group. (b) Representative $I_{\mathrm{Ca}-\mathrm{L}}$ traces recorded from the TAC group. (c) Representative $I_{\mathrm{Ca}-\mathrm{L}}$ traces recorded from the TAC group in the presence of $5 \mathrm{~g} / \mathrm{L} \mathrm{WXKL}$. (d) The peak current density-voltage relationship showed that the current densities in the TAC group were significantly increased by a range of $-10 \mathrm{mV}$ to $+10 \mathrm{mV}$, and the current densities in the TAC group after treatment with $5 \mathrm{~g} / \mathrm{L}$ WXKL were significantly reduced by a range of $-10 \mathrm{mV}$ to $+40 \mathrm{mV}$. (e) The peak current densities in each group exhibited significant differences. ((f) and (g)) Representative $I_{\text {Ca-L }}$ traces recorded from the control group and after treatment with $5 \mathrm{~g} / \mathrm{L}$ WXKL. ${ }^{*} P<0.05$ and ${ }^{* *} P<0.01$ versus the control group. ${ }^{\Delta} P<0.05$ and ${ }^{\Delta} P<0.01$ versus the TAC group.

with $5 \mathrm{~g} / \mathrm{L}$ WXKL shifted the $V_{1 / 2 \text {,act }}$ from $13.55 \pm 1.55 \mathrm{mV}$ to $12.50 \pm 1.57 \mathrm{mV}$ in the TAC group $(n=10, P>0.05$, Figure $5(\mathrm{~b}))$. The slope factor $\left(k_{\text {act }}\right)$ activation values in the control group, the TAC group, and the TAC group after treatment with $5 \mathrm{~g} / \mathrm{L}$ WXKL were $18.22 \pm 1.04 \mathrm{mV}, 19.89 \pm$ $1.79 \mathrm{mV}$, and $20.39 \pm 1.96 \mathrm{mV}$, respectively. These values were not significantly different $(n=10, P>0.05$, Figure $5(\mathrm{c})$ ).

However, compared with the control group, the steadystate inactivation curve in the TAC group shifted to a more positive potential. In the presence of $5 \mathrm{~g} / \mathrm{L}$ WXKL, the steadystate inactivation curve of the TAC group was shifted to a more negative potential (Figure $5(\mathrm{~d})$ ). The half-inactivation potentials $\left(V_{1 / 2 \text {,inact }}\right.$, at which $50 \%$ of the channels are inactivated) in the control and the TAC groups were shifted from
$-11.60 \pm 1.15 \mathrm{mV}$ and $-3.83 \pm 0.66 \mathrm{mV}$, respectively $(n=10$, $P<0.01$, Figure 5(e)). The half-inactivation potential in the TAC group after treatment with $5 \mathrm{~g} / \mathrm{L}$ WXKL was $-16.89 \pm$ $2.24 \mathrm{mV}(n=15, P<0.01$, Figure 5(e)). The slope factor $\left(k_{\text {inact }}\right)$ inactivation values in the control group, the TAC group, and the TAC group after treatment with $5 \mathrm{~g} / \mathrm{L}$ WXKL were $9.89 \pm 0.98 \mathrm{mV}, 11.81 \pm 0.38 \mathrm{mV}$, and $13.55 \pm 1.11 \mathrm{mV}$, respectively $(n=15, P<0.01$, Figure $5(\mathrm{f}))$. These results revealed that WXKL reduced the current by accelerating inactivation of the channels.

3.6. Effects of WXKL on the Recovery of $I_{C a-L}$ from Inactivation in the TAC Group. The time course of recovery from inactivation of $I_{\mathrm{Ca}-\mathrm{L}}$ was evaluated using a paired-pulse protocol: 


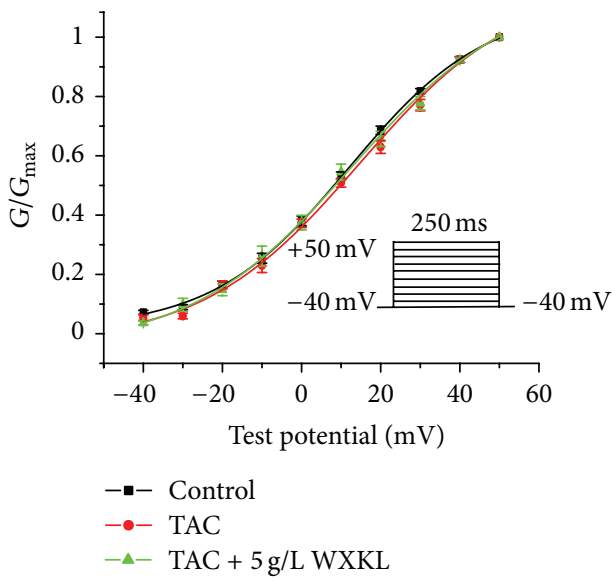

(a)

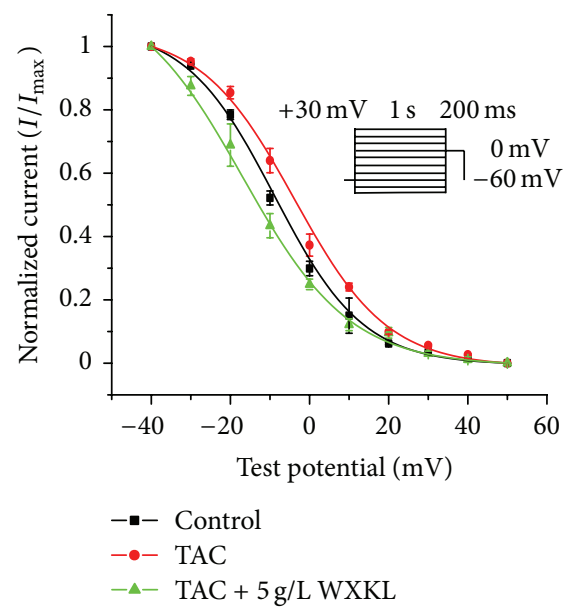

(d)

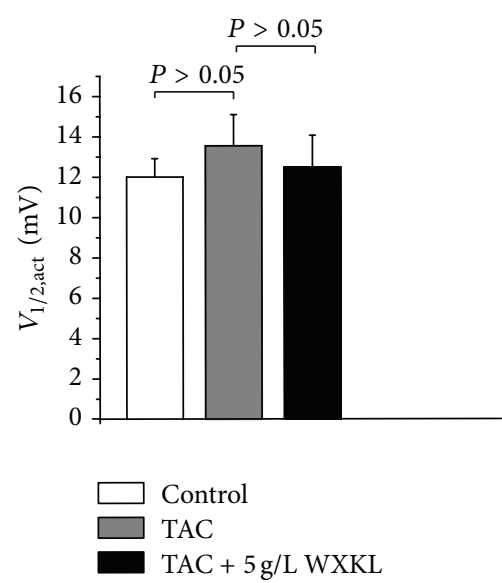

(b)

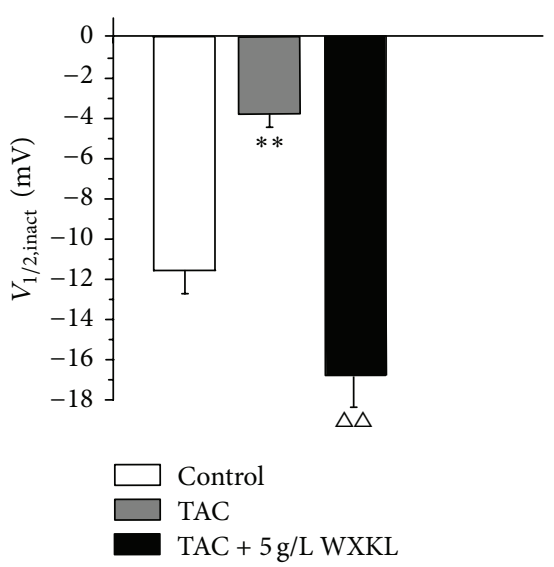

(e)

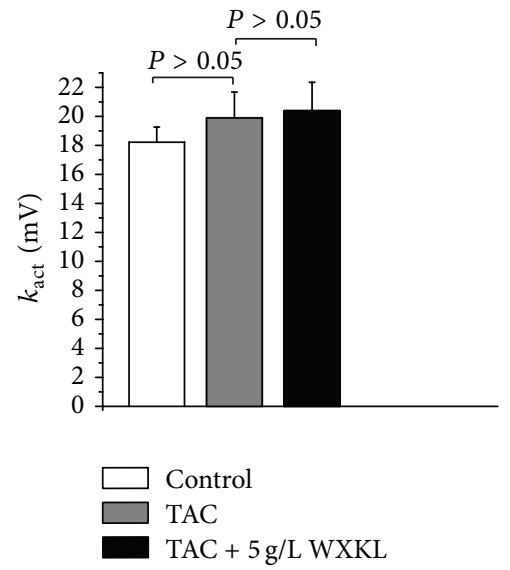

(c)

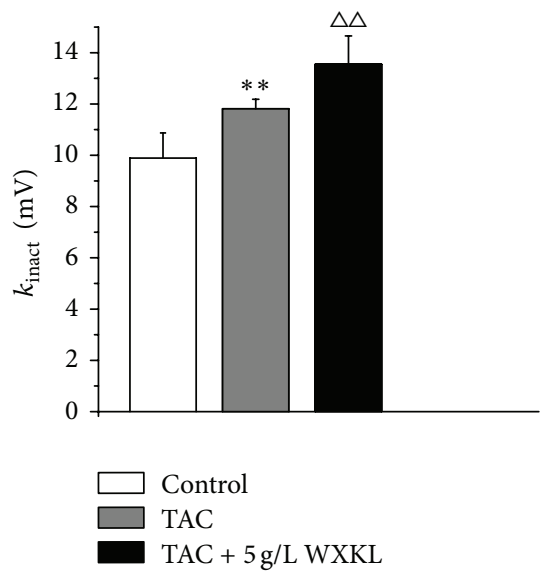

(f)

FIGURE 5: Effects of WXKL on the steady-state activation and inactivation kinetics of the $I_{\mathrm{Ca}-\mathrm{L}}$ in the TAC group. (a) Steady-state activated curve of the control group, the TAC group and the TAC group treated with $5 \mathrm{~g} / \mathrm{L}$ WXKL. The steady-state activation curve of each group did not exhibit significant differences. (b) The $V_{1 / 2 \text {,act }}$ of each group did not exhibit significant differences. (c) The $k_{\text {act }}$ of each group did not exhibit significant differences. (d) The steady-state inactivation curve of the TAC group was shifted to a more negative potential, whereas the steady-state inactivation curve of the TAC group treated with $5 \mathrm{~g} / \mathrm{L} \mathrm{WXKL}$ was shifted to a more active potential. (e) The $V_{1 / 2 \text {,inact }}$ in each group exhibited significant differences. (f) The $k_{\text {inact }}$ of each group exhibited significant differences. ${ }^{*} P<0.05$ and ${ }^{* *} P<0.01$ versus the control group. ${ }^{\Delta} P<0.05$ and ${ }^{\Delta \Delta} P<0.01$ versus the TAC group.

a conditioning pulse was first applied from a holding potential of $-80 \mathrm{mV}$ to $0 \mathrm{mV}$, and a test potential of $0 \mathrm{mV}$ was then applied for $250 \mathrm{~ms}$ after various interval durations of $0.05,0.1$, $0.5,1,5,10,20,40,80,160,320,640,1280,2560$, and $5120 \mathrm{~ms}$. The recovery curve from inactivation was fitted by a single exponential function. The treatment with $5 \mathrm{~g} / \mathrm{L}$ WXKL shifted the recovery curve from the inactivation of $I_{\mathrm{Ca}-\mathrm{L}}$ in the TAC group to the right (Figures 6(a) and 6(b)). The results showed that WXKL delayed the recovery time from inactivation.

\section{Discussion}

We can draw the following conclusions from the present study: (1) in the TAC group, WXKL treatment can significantly decrease the prolongation of the APD in a dosedependent manner, and the $\mathrm{APD}_{20}, \mathrm{APD}_{50}$, and $\mathrm{APD}_{90}$ were all significantly shortened. (2) The amplitude of the $I_{\mathrm{Ca}-\mathrm{L}}$ in the TAC group was increased compared with the control group, and WXKL treatment significantly reduced the $I_{\mathrm{Ca}-\mathrm{L}}$ in the TAC group. (3) WXKL decreased the $I_{\mathrm{Ca}-\mathrm{L}}$ by accelerating the inactivation process of the channels and delaying the recovery time from inactivation but had no significant effect on the activation process. These major findings suggest that $I_{\text {Ca-L }}$ may be the target of the antiarrhythmic effect of WXKL.

Cardiac hypertrophy is a common pathological change that increases the incidence and mortality of many cardiovascular diseases. These changes are frequently induced by electrical remodelling and arrhythmogenesis. Most of the drugs that have been used can potentially induce ventricular arrhythmias. Thus, it is necessary to identify more effective and safer drugs for the treatment of arrhythmias induced by cardiac hypertrophy.

The functioning of the heart depends on the normal action potential, and the normal action potential depends 


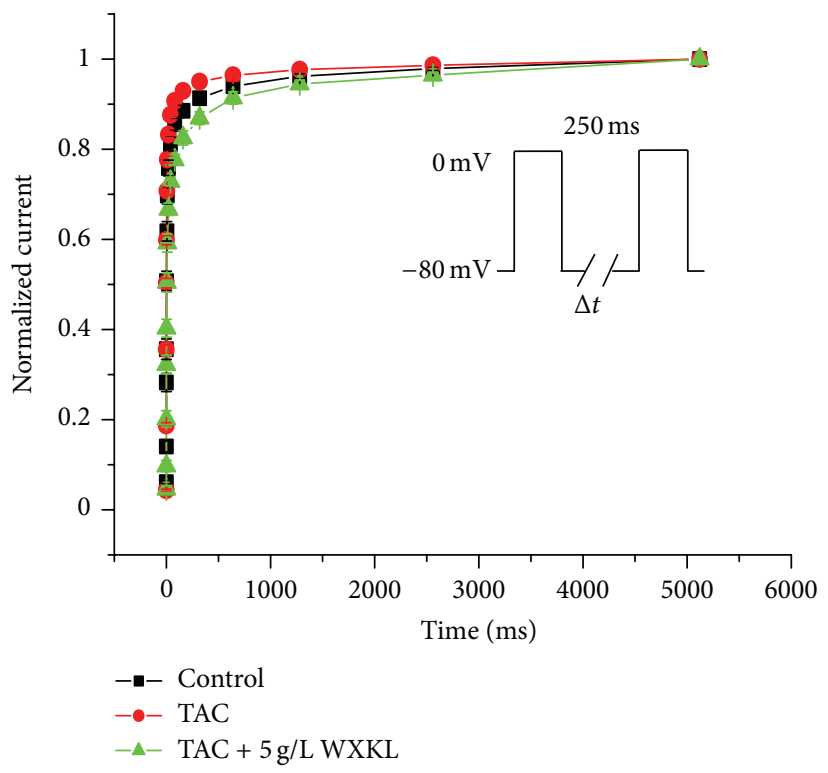

(a)

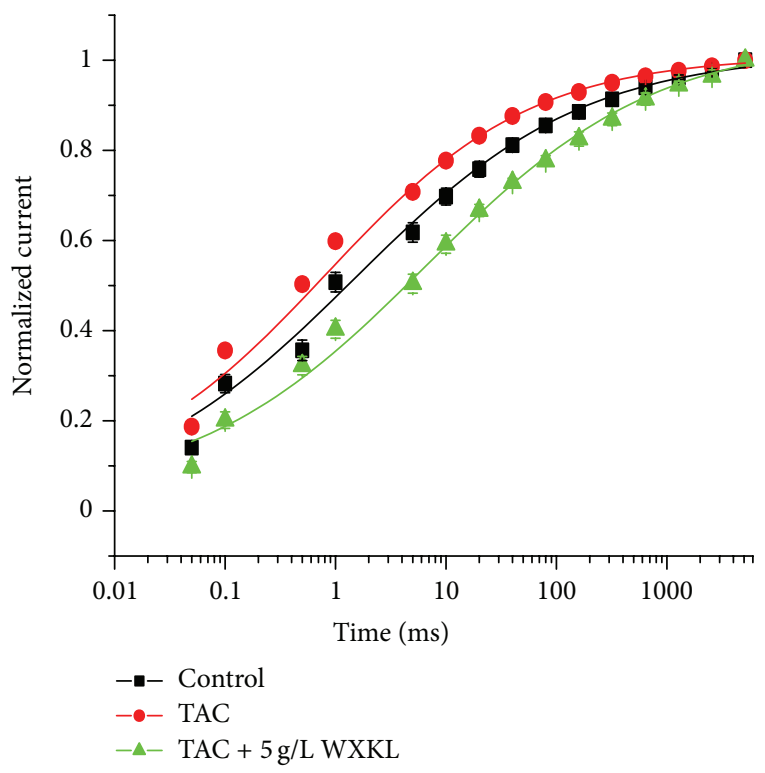

(b)

FIGURE 6: Effects of WXKL on the recovery of the $I_{\mathrm{Ca}-\mathrm{L}}$ from inactivation in the TAC group. The time course of the recovery from inactivation was fitted with a single exponential function. The recovery from inactivation of the $I_{\text {Ca-L }}$ was changed after exposure to $5 \mathrm{~g} / \mathrm{L}$ WXKL.

on the normal functioning of ion channels. The abnormality that is most consistently found in animal models of cardiac hypertrophy is the prolongation of the APD [23-25]. $\mathrm{Ca}^{2+}$ plays a key role in the excitation-contraction coupling and the activation of $\mathrm{Ca}^{2+}$-dependent signalling pathways. The APD prolongation may increase the $\mathrm{Ca}^{2+}$ entry via $I_{\text {Ca-L }}$ during the long plateau phase, which would cause an accumulation of $\mathrm{Ca}^{2+}$ in the sarcoplasmic reticulum (SR) and spontaneous $\mathrm{SR} \mathrm{Ca}^{2+}$ release [26]. Increases in the intracellular $\mathrm{Ca}^{2+}$ concentration, which can be caused by multiple mechanisms, such as L-type $\mathrm{Ca}^{2+}$ channels coupled with $\mathrm{Ca}^{2+}$-induced $\mathrm{Ca}^{2+}$ release from the ryanodine receptors, and T-type $\mathrm{Ca}^{2+}$ channels could lead to cardiac hypertrophy [27]. However, the L-type $\mathrm{Ca}^{2+}$ channel is involved in the predominant mechanism responsible for the influx of $\mathrm{Ca}^{2+}$ in cardiac cells [28]. This channel also plays an important role in the generation of AP under physiological and pathophysiological conditions. The blockade of the L-type $\mathrm{Ca}^{2+}$ channels results in antiarrhythmic actions [29]. Thus, blocking the $\mathrm{Ca}^{2+}$ channels, reducing the $\mathrm{Ca}^{2+}$ overload; and weakening the myocardial contractility will benefit the treatment of cardiac hypertrophy and heart failure.

WXKL is the first antiarrhythmic Chinese medicine to be approved by the state. A large number of clinical trials have confirmed that WXKL can improve the left ventricular diastolic function and reduce the degree of left ventricular hypertrophy with high blood pressure, which ultimately leads to a reduction in the incidence of arrhythmias [30]. Most of the previous studies that analysed the antiarrhythmic properties of WXKL were based on normal myocytes or on drug-induced cardiac hypertrophy. However, it was unknown whether the drugs would exhibit the same effect on pathological myocytes, and the experimental studies on this subject were rare. During these years, an increasing number of studies have been performed using animal models of hypertrophy. A large number of studies have been performed in rats using different interventions to induce hypertrophy [31, 32]. Thus, this paper investigated the antiarrhythmic effects of WXKL on a TAC model using an electrophysiological technology.

Previous experiments have shown that WXKL can reverse cardiac hypertrophy induced by ISO. WXKL significantly reduced $\mathrm{HW} / \mathrm{BW}, \mathrm{LVW} / \mathrm{BW}$ and the expression of $\beta$-catenin and e-myc. Thus, the use of WXKL for the treatment of patients with hypertension and arrhythmia may be a reasonable and effective choice [33]. Our previous study has shown that WXKL inhibits heart failure and cardiac arrhythmias via a mechanism that may involve the regulation of the CaMKII signal transduction pathway similar to amiodarone. WXKL treatment can increase the calcium transient amplitude in isolated cardiac myocytes from rats with myocardial infarction and reduce the incidence of cardiac arrhythmias in rat myocardial infarction model [34]. In our model, we found that the APD of the TAC group was significantly prolonged compared with the control group, which was in accordance with the results of previous studies $[25,35]$. The $\mathrm{APD}_{20}, \mathrm{APD}_{50}$, and $\mathrm{APD}_{90}$ were all longer than those of the control group. After WXKL treatment, the $\mathrm{APD}_{20}, \mathrm{APD}_{50}$, and $\mathrm{APD}_{90}$ of the TAC group were significantly shortened. WXKL abbreviated the prolongation of the APD in a dosedependent manner. The change in the APD indicated that the ion channel currents of the TAC group were also changed. Considering the important role of $\mathrm{Ca}^{2+}$ in cardiac hypertrophy, we investigated the effect of WXKL on the L-type $\mathrm{Ca}^{2+}$ channel.

A previous study showed the effect of WXKL on the $I_{\mathrm{Ca}-\mathrm{L}}$ and $I_{\text {to }}$ in normal rat ventricular myocytes. WXKL decreased the $I_{\mathrm{Ca}-\mathrm{L}}$, shifted the steady-state activation curve to the 
right, and prolonged the recovery time from inactivation [17]. However, the results from studies of changes of the $I_{\text {Ca-L }}$ in cardiac hypertrophy models are inconsistent. These disparities are due in part to the differences in the models used and the variations in the experimental conditions. Our results are consistent with the studies that showed a significant increase in the $\mathrm{Ca}^{2+}$ current [24]. In our study, we found that both the current amplitude and the current density of the $I_{\mathrm{Ca}-\mathrm{L}}$ in the TAC group were higher than those in the control group. The acute application of WXKL inhibited the $I_{\mathrm{Ca}-\mathrm{L}}$ in a concentration-dependent manner in the control group. The $\mathrm{IC}_{50}$ was found to be $6.23 \mathrm{~g} / \mathrm{L}$. WXKL significantly decreased the peak current of the $I_{\mathrm{Ca}-\mathrm{L}}$ in the TAC group. It appears that the effect of WXKL can significantly relieve the increase in the $I_{\mathrm{Ca}-\mathrm{L}}$ in the TAC group. The experiments revealed that WXKL was able to block the $I_{\mathrm{Ca}-\mathrm{L}}$, which may account for the shortening of the APD and contribute to some of its antiarrhythmic effects. WXKL significantly reduced the $\mathrm{APD}_{20}, \mathrm{APD}_{50}$, and $\mathrm{APD}_{90}$ of the TAC group. This result is well explained by the reduction of the $I_{\mathrm{Ca}-\mathrm{L}}$. We demonstrated that WXKL has the potential to attenuate the development of cardiac hypertrophy by affecting the signalling mechanisms of cardiac myocytes. In our study, a WXKL concentration of $5 \mathrm{~g} / \mathrm{L}$ was used to explore the mechanism through which this agent treats cardiac hypertrophy. This concentration is close to the $\mathrm{IC}_{50}$. However, the results show that the WXKL treatment decreased the $I_{\mathrm{Ca}-\mathrm{L}}$ in the TAC group and in the control group to a level that was lower than the normal level. We should therefore use a lower dose of WXKL and analyse the resulting effects on the $I_{\mathrm{Ca}-\mathrm{L}}$ of the TAC group. In addition, it is unclear whether an excessive dose of WXKL can exert a significant antiarrhythmic effect with fewer side effects.

Furthermore, we determined the effect of WXKL on the gating mechanism of $I_{\mathrm{Ca}-\mathrm{L}}$ in the TAC group. The steadystate activation curves in each group were not significantly different. Compared with the control group, the steady-state inactivation curve of the TAC group was shifted to a more positive potential. In the presence of $5 \mathrm{~g} / \mathrm{L}$ WXKL, the steadystate activation curve was shifted to a more negative potential. This result suggests that the voltage-dependent steady-state inactivation of the L-type $\mathrm{Ca}^{2+}$ channels was accelerated. Moreover, $5 \mathrm{~g} / \mathrm{L}$ WXKL shifted the recovery curve from inactivation of the $I_{\mathrm{Ca}-\mathrm{L}}$ in the TAC group to the right. These data suggested that WXKL decreased the $I_{\text {Ca-L }}$ through facilitation of the steady-state inactivation and retardation of the recovery from inactivation. Interestingly, we found that the effects of WXKL on the steady-state activation and inactivation procedures of the L-type $\mathrm{Ca}^{2+}$ channels in the TAC group were not in accordance with its effects on normal rats [17]. An explanation for this discrepancy is that pathological cells may have undergone electrical remodelling, which would change the effect that WXKL would have on these cells. The effects of WXKL on the recovery curve from inactivation of $I_{\mathrm{Ca}-\mathrm{L}}$ were consistent and showed that the mechanism underlying the beneficial effects of WXKL may involve the regulation of the $\mathrm{Ca}^{2+}$ channel and a reduction in the $\mathrm{Ca}^{2+}$ influx. WXKL likely affects the L-type $\mathrm{Ca}^{2+}$ channels, alters the cellular $\mathrm{Ca}^{2+}$ regulation, and improves the heart function.
WXKL includes five ingredients: Nardostachys chinensis Batal extract, codonopsis, notoginseng, amber, and Rhizoma Polygonati. A study had investigated the effects of Nardostachys chinensis Batal extract $(\mathrm{NcBe})$ on the activation kinetics of normal rat cardiac sodium channels and transient outward potassium channels. NcBe significantly blocks the $I_{\mathrm{Na}}$ and $I_{\text {to }}$ of normal rat ventricular myocytes [36]. Studies could be conducted to discover more effective drugs. Furthermore, the present study focused on the effect of WXKL in the $I_{\mathrm{Ca}-\mathrm{L}}$ of TAC rats. To investigate the mechanism through which WXKL exerts antiarrhythmic effect, it is necessary to study the related signal transduction pathways in further study. In addition to the shortening of the APD after WXKL perfusion and the excessive reduction of the $I_{\mathrm{Ca}-\mathrm{L}}$, other ion currents in hypertrophied myocytes may be modulated by this herbal extract. Therefore, it will be valuable to obtain further insight into the effect of WXKL on the regulation of other ion channels and the related signal transduction pathway.

In summary, the present study evaluated the electrophysiologic effects and antiarrhythmic potential of WXKL in hypertrophied ventricular myocytes. The results demonstrated that WXKL treats cardiac hypertrophy and cardiac arrhythmias via a mechanism that may involve the regulation of the L-type $\mathrm{Ca}^{2+}$ channels. WXKL treatment significantly shortened the prolongation of the APD and reduced the $I_{\mathrm{Ca}-\mathrm{L}}$. Further studies should explore the deeper mechanism through which WXKL treats cardiac hypertrophy to ultimately offer new avenues for the prevention and treatment of this and other related diseases.

\section{Conflict of Interests}

All authors declare that they have no conflict of interests.

\section{Author's Contribution}

Yu Chen, Yang Li, Lili Guo, and Wen Chen contributed equally to this paper.

\section{Acknowledgments}

This work was supported by the National Natural Science Foundation of China (Grant no. 81001514 and no. 81373835), the Beijing Nova Program (Grant no. 2011110), and the Fundamental Research Funds for the Central Public Welfare Research Institutes, (Grant no. ZZ070802).

\section{References}

[1] V. L. Roger, A. S. Go, D. M. Lloyd-Jones et al., "Executive summary: heart disease and stroke statistics-2012 update: a report from the American heart association," Circulation, vol. 125, no. 1, pp. 188-197, 2012.

[2] B. J. Maron, J. J. Doerer, T. S. Haas, D. M. Tierney, and F. O. Mueller, "Sudden deaths in young competitive athletes analysis of 1866 deaths in the united states, 1980-2006," Circulation, vol. 119, no. 8, pp. 1085-1092, 2009. 
[3] D. M. Bers, "Calcium cycling and signaling in cardiac myocytes," Annual Review of Physiology, vol. 70, pp. 23-49, 2008.

[4] D. A. Eisner, T. Kashimura, L. A. Venetucci, and A. W. Trafford, "From the ryanodine receptor to cardiac arrhythmias," Circulation Journal, vol. 73, no. 9, pp. 1561-1567, 2009.

[5] L. D. Gauthier, J. L. Greenstein, and R. L. Winslow, “Toward an integrative computational model of the Guinea pig cardiac myocyte," Frontiers in Physiology, vol. 3, no. 244, pp. 1-19, 2012.

[6] E. M. Cherry, F. H. Fenton, and R. F. Jr. Gilmour, "Mechanisms of ventricular arrhythmias: a dynamical systems-based perspective," American Journal of Physiology, vol. 302, no. 12, pp. H2451H2463, 2012.

[7] R. Rouet, M. E. Worou, P. E. Puddu et al., "Nifedipine blocks ondansetron electrophysiological effects in rabbit purkinje fibers and decreases early afterdepolarization incidence," Current Clinical Pharmacology, vol. 7, no. 1, pp. 41-48, 2012.

[8] E. F. Aziz, F. Javed, B. Pratap, and E. Herzog, "Strategies for the prevention and treatment of sudden cardiac death," Journal of Open Access Emergency Medicine, vol. 2010, no. 2, pp. 99-114, 2010.

[9] E. J. Velazquez, J. B. Williams, E. Yow et al., "Long-term survival of patients with ischemic cardiomyopathy treated by coronary artery bypass grafting versus medical therapy," Annals of Thoracic Surgery, vol. 93, no. 2, pp. 523-530, 2012.

[10] J. Kalifa and U. M. R. Avula, “The Chinese herb extract Wenxin Keli: atrial selectivity from the Far East," Heart Rhythm, vol. 9, no. 1, pp. 132-133, 2012.

[11] H.-Y. Sun, "Clinical study of Wenxikeli treatment of viral myocarditis," Zhonghua Shi Yan He Lin Chuang Bing Du Xue Za Zhi, vol. 23, no. 2, pp. 144-145, 2009.

[12] P.-Y. Xie and S.-H. Shen, "Effect of combination of Chinese and Western medicines on sinus rhythm maintenance in patients with auricular fibrillation after conversion," Zhongguo Zhong Xi Yi Jie He Za Zhi Zhongguo Zhongxiyi Jiehe Zazhi, vol. 26, no. 7, pp. 644-646, 2006.

[13] M. Wang, Y.-B. Yu, and S.-E. Huang, "Clinical observation on effect and safety of combined use of wenxin granule and amiodarone for conversion of auricular fibrillation," Zhongguo Zhong Xi Yi Jie He Za Zhi Zhongguo Zhongxiyi Jiehe Zazhi, vol. 26, no. 5, pp. 445-448, 2006.

[14] A. Burashnikov, A. Petroski, D. Hu, H. Barajas-Martinez, and C. Antzelevitch, "Atrial-selective inhibition of sodium-channel current by Wenxin Keli is effective in suppressing atrial fibrillation," Heart Rhythm, vol. 9, no. 1, pp. 125-131, 2012.

[15] J. M. Zhou, Z. G. Ye, X. M. Cui, Z. Jing A Zhao, and L. Zhu, "Pharmacodynamic research of notoginsenoside R1, R2 and ginsenoside Rb1," Chinese Traditional Patent Medicine, vol. 32, no. 9, pp. 1494-1497, 2010.

[16] F. Zhou, S.-J. Hu, and Y. Mu, "Protection effect of Wenxin Keli on isoproterenol induced heart failure in rats," Zhongguo Zhongyao Zazhi, vol. 32, no. 16, pp. 1676-1679, 2007.

[17] X. Wang, X. Wang, Y. Gu, T. Wang, and C. Huang, "Wenxin Keli attenuates ischemia-induced ventricular arrhythmias in rats: involvement of L-type calcium and transient outward potassium currents," Molecular Medicine Reports, vol. 7, no. 2, pp. 519-524, 2013.

[18] Y.-M. Lu, J. Huang, N. Shioda et al., "Camkiißb mediates aberrant NCX1 expression and the imbalance of NCX1/SERCA in transverse aortic Constriction-Induced failing heart," PLoS ONE, vol. 6, no. 9, Article ID e24724, 2011.
[19] Y.-M. Lu, N. Shioda, F. Han et al., "Imbalance between CaM kinase II and calcineurin activities impairs caffeine-induced calcium release in hypertrophic cardiomyocytes," Biochemical Pharmacology, vol. 74, no. 12, pp. 1727-1737, 2007.

[20] J. Wang, N. Xu, X. Feng et al., "Targeted disruption of Smad4 in cardiomyocytes results in cardiac hypertrophy and heart failure," Circulation Research, vol. 97, no. 8, pp. 821-828, 2005.

[21] J. Tytgat, "How to isolate cardiac myocytes," Cardiovascular Research, vol. 28, no. 2, pp. 280-283, 1994.

[22] F. Han, Y.-M. Lu, H. Hasegawa et al., "Inhibition of dystrophin breakdown and endothelial nitric-oxide synthase uncoupling accounts for cytoprotection by 3-[2-[4-(3-chloro-2-methylphenyl)-1- piperazinyl]ethyl]-5,6-dimethoxy-1-(4-imidazolylmethyl)-1H-indazole dihydrochloride 3.5 hydrate (DY-9760e) in left ventricular hypertrophied mice," Journal of Pharmacology and Experimental Therapeutics, vol. 332, no. 2, pp. 421-428, 2010.

[23] M. E. Díaz, H. K. Graham, and A. W. Trafford, "Enhanced sarcolemmal $\mathrm{Ca}^{2+}$ efflux reduces sarcoplasmic reticulum $\mathrm{Ca}^{2+}$ content and systolic $\mathrm{Ca}^{2+}$ in cardiac hypertrophy," Cardiovascular Research, vol. 62, no. 3, pp. 538-547, 2004.

[24] W. U. Foltz, M. Wagner, E. Rudakova, and T. Volk, "N-acetylcysteine prevents electrical remodeling and attenuates cellular hypertrophy in epicardial myocytes of rats with ascending aortic stenosis," Basic Research in Cardiology, vol. 107, no. 5, p. 290, 2012.

[25] C. Shi, X. Wang, F. Dong et al., "Temporal alterations and cellular mechanisms of transmural repolarization during progression of mouse cardiac hypertrophy and failure," Acta Physiologica, vol. 208, no. 1, pp. 95-110, 2013.

[26] P. Milberg, M. Fink, C. Pott et al., "Blockade of ICa suppresses early afterdepolarizations and reduces transmural dispersion of repolarization in a whole heart model of chronic heart failure," British Journal of Pharmacology, vol. 166, no. 2, pp. 557-568, 2012.

[27] J. D. Molkentin, "Dichotomy of $\mathrm{Ca}^{2+}$ in the heart: Contraction versus intracellular signaling," Journal of Clinical Investigation, vol. 116, no. 3, pp. 623-626, 2006.

[28] G. M. Faber, J. Silva, L. Livshitz, and Y. Rudy, "Kinetic properties of the cardiac L-type $\mathrm{Ca}^{2+}$ channel and its role in myocyte electrophysiology: a theoretical investigation," Biophysical Journal, vol. 92, no. 5, pp. 1522-1543, 2007.

[29] D. J. Triggle, "Calcium channel antagonists: clinical uses-past, present and future," Biochemical Pharmacology, vol. 74, no. 1, pp. 1-9, 2007.

[30] N. Su, T. Xu, Z. Zhou, and Y. Tang, "Efficacy and safety of wenxin granules in the treatment of congestive heart failure: a systematic review," China Pharmacy, vol. 21, no. 7, pp. 637-640, 2010.

[31] G. Hasenfuss, "Animal models of human cardiovascular disease, heart failure and hypertrophy," Cardiovascular Research, vol. 39, no. 1, pp. 60-76, 1998.

[32] Z. Abassi, I. Goltsman, T. Karram, J. Winaver, and A. Hoffman, "Aortocaval fistula in rat: a unique model of volume-overload congestive heart failure and cardiac hypertrophy," Journal of Biomedicine and Biotechnology, vol. 2011, Article ID 729497, 13 pages, 2011.

[33] G. Q. Wang, W. Jin, M. Y. Qi et al., "The mechanism of the effects of Wenxinkeli on cardiac hypertrophy in rats," Chinese Journal of Integrative Medicine on Cardio-/Cerebrovascular Disease, vol. 9, no. 4, pp. 462-463, 2011.

[34] Y. W. Xing, Y. H. Gao, J. X. Chen et al., "Wenxin-keli regulates the calcium/calmodulin-dependent Protein kinase II Signal 
transduction pathway and inhibits cardiac arrhythmia in rats with myocardial infarction," Evidence-Based Complementary and Alternative Medicine, vol. 2013, Article ID 464508, 15 pages, 2013.

[35] C. X. Shi, Y. H. Wang, F. Dong, Y. J. Zhang, and Y. F. Xu, "Transmural L-type calcium current in a pressure-overloaded mouse model with heart failure," Sheng Li Xue Bao, vol. 59, no. 1, pp. 19-26, 2007.

[36] Y. W. Liu, J. H. Guo, P. Zhang, J. W. Li, and C. Li, "The effects of Nardostachys chinensis batal extract on the sodium current and transient outward potassium current of rat ventricular myocytes," Chinese Journal of Cardiac Pacing and Electrophysiology, vol. 23, no. 6, pp. 533-535, 2009. 


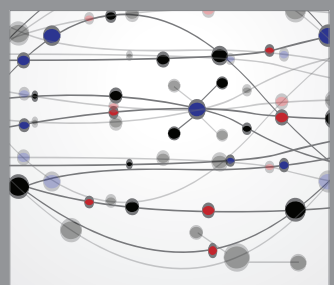

The Scientific World Journal
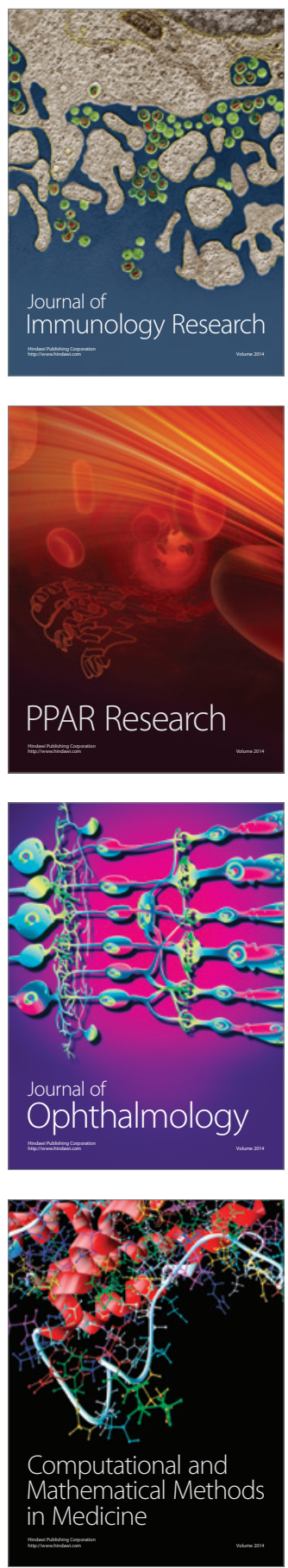

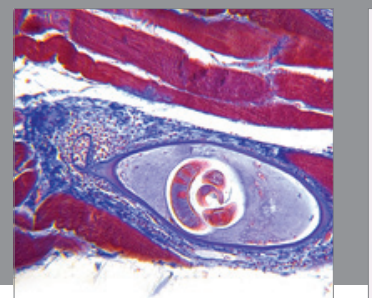

Gastroenterology

Research and Practice
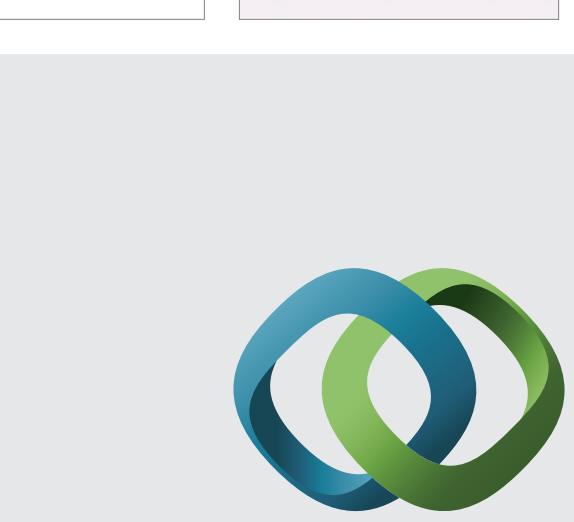

\section{Hindawi}

Submit your manuscripts at

http://www.hindawi.com
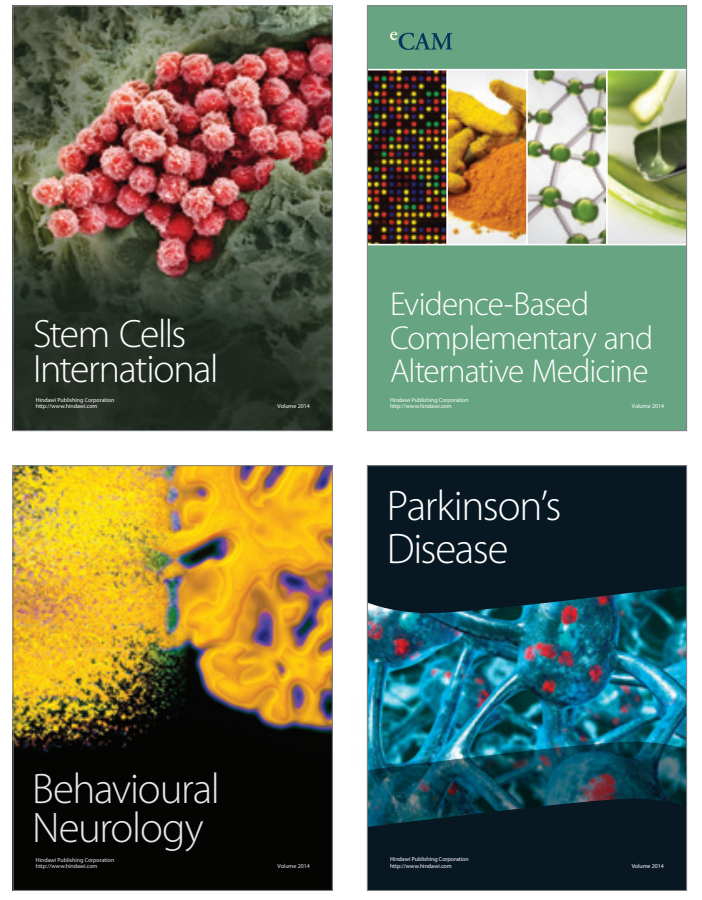
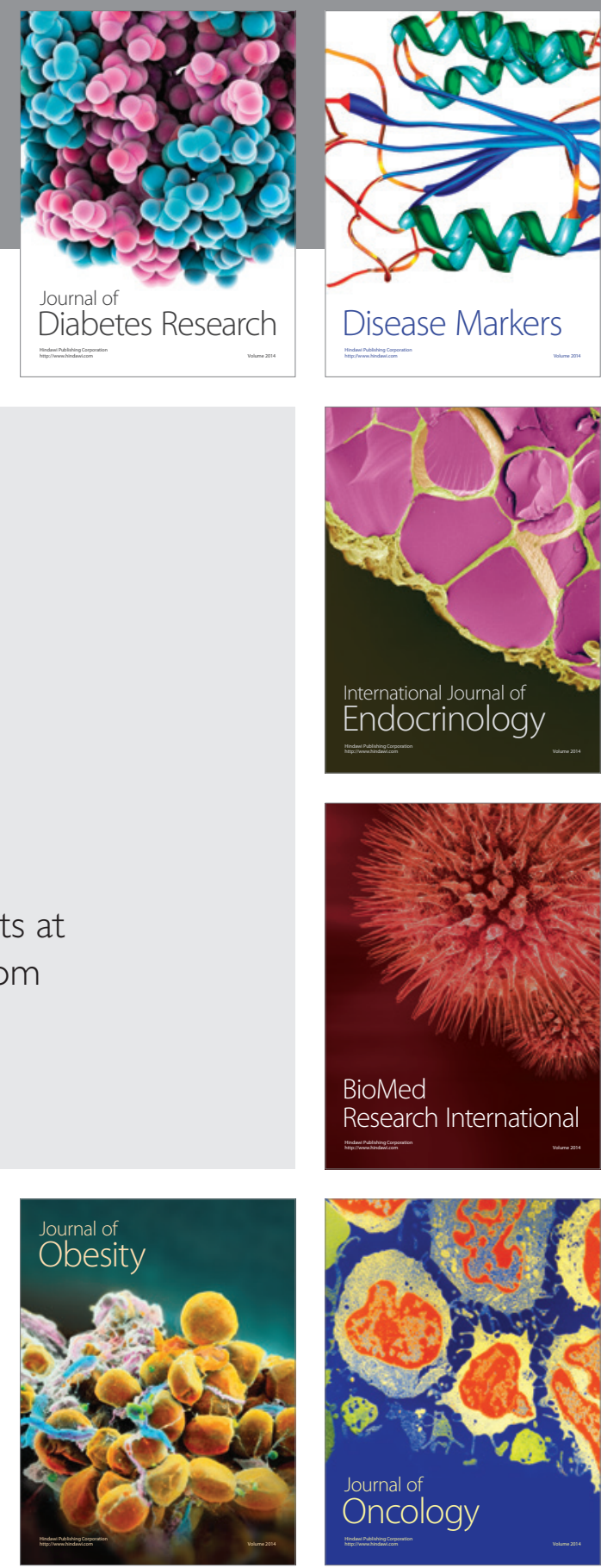

Disease Markers
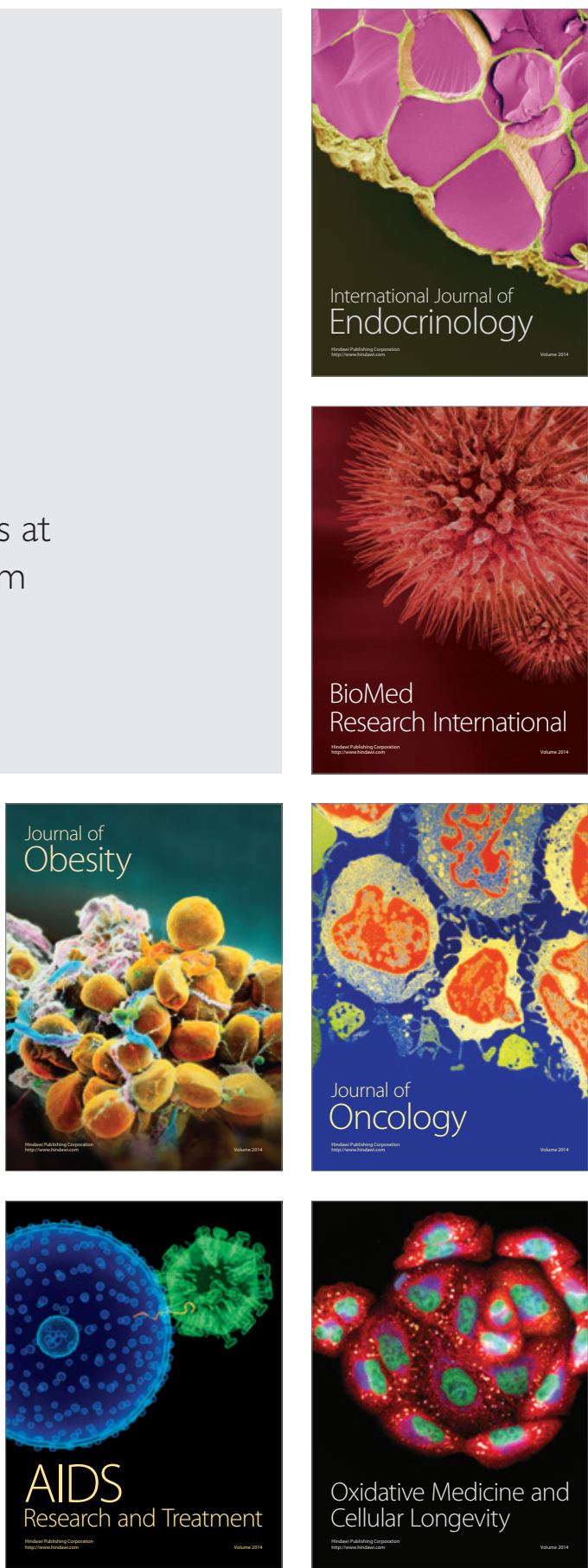